\title{
Development of Pyridine-based Inhibitors for the Human Vaccinia- related Kinases 1 and 2
}

Ricardo A. M. Serafim, ${ }^{\dagger,}, \#$ Fernando H. de Souza Gama, ${ }^{\S, \#}$ Luiz A. Dutra, ${ }^{\dagger,}, \#$ Caio V. dos Reis, ${ }^{\dagger, \ddagger}$ Stanley N. S. Vasconcelos, ${ }^{\dagger, \ddagger}$ André da Silva Santiago, ${ }^{\dagger, \$}$ Jéssica E. Takarada, ${ }^{\dagger, \ddagger}$ Fúlvia Di Pillo, Hatylas Azevedo, ${ }^{\S}$ Alessandra Mascarello, ${ }^{\S}$ Jonathan M. Elkins, ${ }^{\ddagger}, \perp$ Katlin B. Massirer, ${ }^{\dagger,}$ Opher Gileadi, ${ }^{\perp}$ Cristiano R. W. Guimarães, ${ }^{*}, \S$ and Rafael M. Couñago $*, \dagger, \ddagger$ (i)

${ }^{\dagger}$ Centro de Química Medicinal (CQMED), Centro de Biologia Molecular e Engenharia Genética (CBMEG), Universidade Estadual de Campinas (UNICAMP), Campinas, SP 13083-875, Brazil

${ }^{\ddagger}$ Structural Genomics Consortium, Departamento de Genética e Evolução, Instituto de Biologia, UNICAMP, Campinas, SP 13083-886, Brazil

${ }^{\S}$ Aché Laboratórios Farmacêuticos S.A., Guarulhos, SP, Brazil

"PhD Program in Genetics and Molecular Biology (PGBM), UNICAMP, Campinas, SP 13083-886, Brazil

${ }^{\perp}$ Structural Genomics Consortium, Nuffield Department of Medicine, University of Oxford, Oxford OX3 7DQ U.K.

Supporting Information

ABSTRACT: Vaccinia-related kinases 1 and 2 (VRK1 and VRK2) are human Ser/Thr protein kinases associated with increased cell division and neurological disorders. Nevertheless, the cellular functions of these proteins are not fully understood. Despite their therapeutic potential, there are no potent and specific inhibitors available for VRK1 or VRK2. We report here the discovery and elaboration of an aminopyridine scaffold as a basis for VRK1 and VRK2 inhibitors. The most potent compound for VRK1 (26) displayed an $\mathrm{IC}_{50}$ value of $150 \mathrm{nM}$ and was fairly selective in a panel of 48 human kinases (selectivity score $\mathrm{S}(50 \%)$ of 0.04$)$. Differences in compound binding mode and substituent preferences between the two VRKs were identified by the structure-activity relationship combined with the crystallographic analysis of key compounds. We expect our results to serve as a starting point for the design of more specific and potent inhibitors against each of the two VRKs.

KEYWORDS: Vaccinia-related kinases, pyridine, difluorophenol, kinase inhibitors, structure-based compound development
$\mathrm{T}$ he human genome encodes three vaccinia-related kinases (VRK1, VRK2, and VRK3). ${ }^{1}$ VRK3 has a degraded ATPbinding site and is thought to be a pseudokinase, ${ }^{2}$ while VRK1 and VRK2 feature active kinase domains and have been associated with various cellular processes, including cell replication, chromatin remodeling, and response to DNA damage. $^{3-5}$ VRK2 has two isoforms that share identical kinase domains but locate to distinct cellular compartments: endoplasmic reticulum (VRK2A) and cytoplasm and nucleus (VRK2B). ${ }^{1,6}$ VRK1 can be found in both nucleus and cytoplasm. ${ }^{1}$ Genetic studies have associated VRK1 and VRK2 with a number of human diseases and conditions. VRK2 is associated with neurological disorders, such as schizophrenia, multiple sclerosis, major depressive disorder, and epilepsy. ${ }^{7-11}$ VRK1 overexpression is associated with increased cell division and poor prognosis in a number of cancers. ${ }^{12,13}$ Despite these advances, our understanding of the cellular roles of human VRKs and their therapeutic potential is

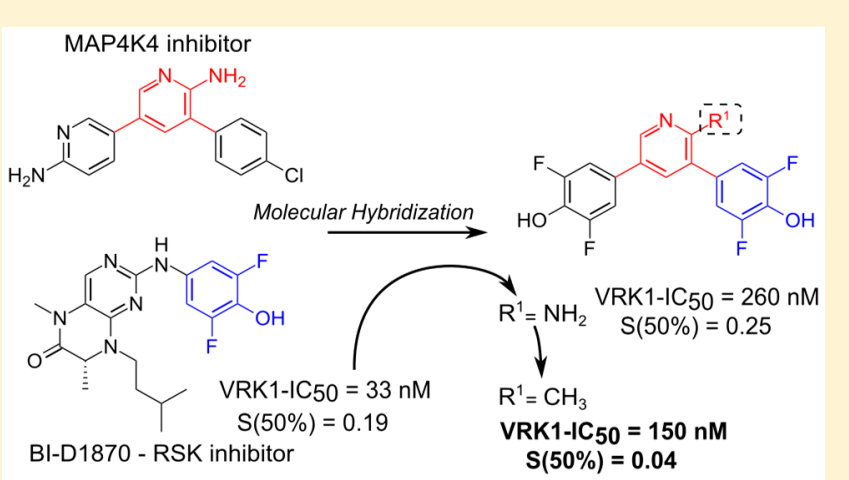

limited. Currently, there are no potent and specific small molecule inhibitors of the VRKs.

Previously, our analysis of the Published Kinase Inhibitor Set (PKIS $)^{14}$ revealed that VRK1 shared a similar activity profile to a small number of kinases, including members of the kinase family Ste20, such as MINK1 and TNIK. ${ }^{15}$ The Ste20 kinase family also includes MAP4K4, whose phosphate-binding loop (P-loop, also referred to as glycine-rich loop) can adopt an unusual conformation in which this conserved structural motif folds over the ligand. It has been suggested that this folded Ploop conformation might be targeted to obtain potent and selective kinase inhibitors. ${ }^{16}$ Cocrystal structures of VRK1 and VRK2 bound to the p90 RSK (ribosomal S6 kinase) inhibitor BI-D1870 (1, Figure 1A) revealed that both kinases can also

Received: March 1, 2019

Accepted: August 19, 2019

Published: August 19, 2019 
(A)

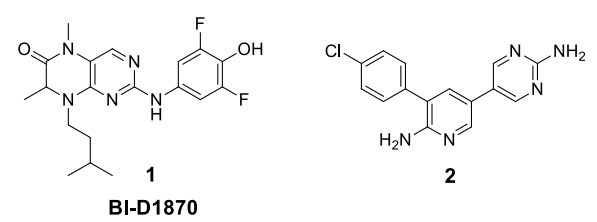

(B)

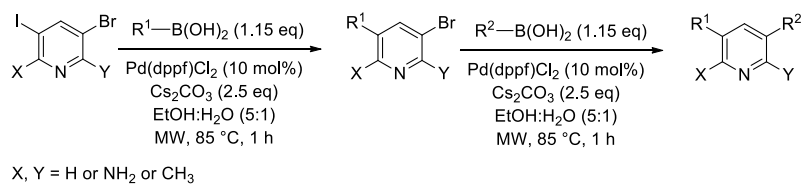

Figure 1. (A) Chemical structure of 1 (BI-D1870) and the MAP4K4 inhibitor 2. (B) Synthetic route to aminopyridine derivatives.

adopt the folded P-loop conformation, further suggesting that MAP4K4 and these two VRKs have similar topography and structural features in their ATP-binding sites. ${ }^{15}$ BI-D1870 displays a highly developed pteridine scaffold, and recent selectivity data revealed this compound to be quite promiscuous. ${ }^{17}$ These observations motivated us to search for MAP4K4 ligands known to induce a folded P-loop conformation, and that could be used as starting points to develop potent and selective inhibitors against VRK1 and VRK2.

2-Aminopyridines are well-known kinase inhibitor scaffolds, and such compounds (exemplified by 2, Figure 1A) have been shown to promote a folded P-loop conformation in MAP4K4. ${ }^{18}$ Additionally, this scaffold is small and versatile, allowing the attachment of different substituents on both sides of the aminopyridine core by a short synthetic route. The cocrystal structures of VRK1 and VRK2 bound to 1 (BID1870) also revealed that the ligand difluorophenol moiety promoted favorable polar interactions to structurally conserved residues within the VRK ATP-binding site. ${ }^{15}$ Thus, our design strategy to develop new small molecule inhibitors for VRK1 and VRK2 was to generate hybrid ligands by fusing the aminopyridine core with the difluorophenol group.

Synthesis of aminopyridine derivatives was performed using a rapid and efficient two-step Suzuki-Miyaura coupling (Figure 1B) with yields ranging between $18 \%$ and $89 \%$. Each step was performed by a microwave-assisted reaction in $1 \mathrm{~h}$. Details of the procedure are available in the Supporting Information.

To test our hybridization hypothesis, we prepared prototype compound 5, bearing difluorophenol moieties in both, the 3and 5-positions of the 2-aminopyridine core (Table 1). We employed a thermal-shift assay (differential scanning fluorimetry, DSF) to identify aminopyridine analogues that could bind to the full-length VRK1 (residues Met1-Lys396, VRK1-FL) and the kinase domain of VRK2 (residues Pro14-His335, VRK2-KD). DSF is a robust method to estimate binding affinities and has been used successfully to triage large compound libraries. ${ }^{14,19,20}$ For these experiments, we considered positive hits those compounds inducing changes in VRK1-FL and VRK2-KD melting temperatures, $\Delta T_{\mathrm{m}}$, larger than $2.0^{\circ} \mathrm{C}$. DSF analysis indicated that $\mathbf{5}$ induced thermal shifts above this threshold for both VRK1 and VRK2 (Table 1, Supplementary Figure S1).

Encouraged by these results, we prepared a first series of 2aminopyridine analogues to explore the relevance of the difluorophenol moiety to the thermal stabilization of the two
Table 1. Structures and Values for DSF and Enzyme Inhibition Assays for Key Compounds

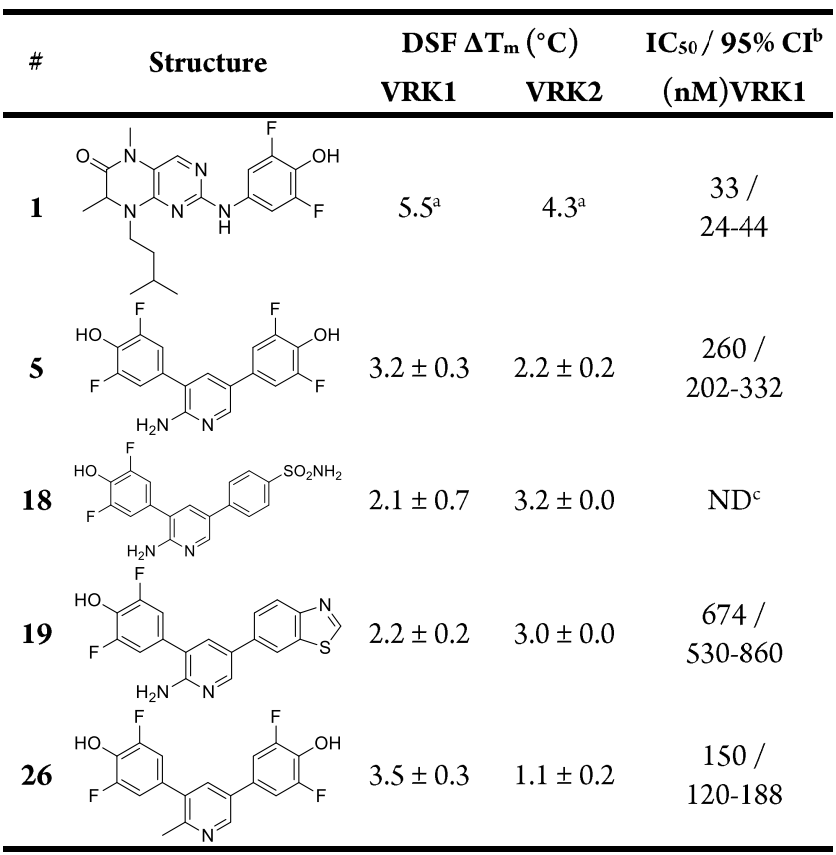

${ }^{a}$ Values from ref $15 .{ }^{b}$ Confidence interval. ${ }^{c}$ Not determined.

VRKs (Table 1, Supplementary Table S1). For both proteins, simultaneously replacing the difluorophenol moiety with phenol groups in $\mathrm{R}^{1}$ and $\mathrm{R}^{2}$ (6) resulted in a drop of the observed $\Delta T_{\mathrm{m}}$, suggesting that increasing the phenol acidic character or altering the electronic properties of the aromatic ring was important for binding. ${ }^{21,22}$ VRK1-FL seemed to tolerate the substitution of a difluorophenol by a phenol on either $\mathrm{R}^{1}$ or $\mathrm{R}^{2}$ position equally well, whereas maintaining a difluorophenol group in $\mathrm{R}^{1}$ proved to be more important for VRK2-KD binding (8 and 7). Not unexpectedly, removal of the $\mathrm{HB}$ (hydrogen bond) capacity in $\mathrm{R}^{2}$, while maintaining the difluorophenol group in $\mathrm{R}^{1}$, still resulted in a $\Delta T_{\mathrm{m}}$ drop for both VRKs (9). We attempted to improve compound binding by replacing the difluorophenol in $\mathrm{R}^{1}$ with a variety of groups of distinct sizes, polarities, and electronic proprieties, but none of these changes resulted in an improved $\Delta T_{\mathrm{m}}$ over the difluorophenol moiety.

We next explored the ability of various phenyl derivatives in $\mathrm{R}^{2}$ to optimize the compound binding profile, while maintaining a difluorophenol group in $\mathrm{R}^{1}$ (Supplementary Table S1). Our results confirmed that polar groups in $\mathrm{R}^{2}$ are important for binding the VRKs and indicated that, overall, these groups were better tolerated in the meta position (10 vs $11 ; 12$ vs $13 ; 14$ vs 15 and 16 ), although the addition of a carboxylic acid group in this position was detrimental to binding (17). For VRK2-KD, the best compound had a parasulfonamide group on the $\mathrm{R}^{2}$ phenyl ring (18), although a benzothiazole fused ring (19) was also tolerated in this position. VRK1-FL tolerated both of these groups in $\mathrm{R}^{2}$ equally well. Nevertheless, for VRK1, we could not find a $R^{2}$ substituent that increased $T_{\mathrm{m}}$-shifts compared to the difluorophenol moiety (5). For VRK2-KD, further attempts to modify the difluorophenol in $\mathrm{R}^{1}$ while maintaining the sulfonamide group in $\mathrm{R}^{2}$ were unsuccessful $(\mathbf{2 0}, \mathbf{2 1}$, and 22). 

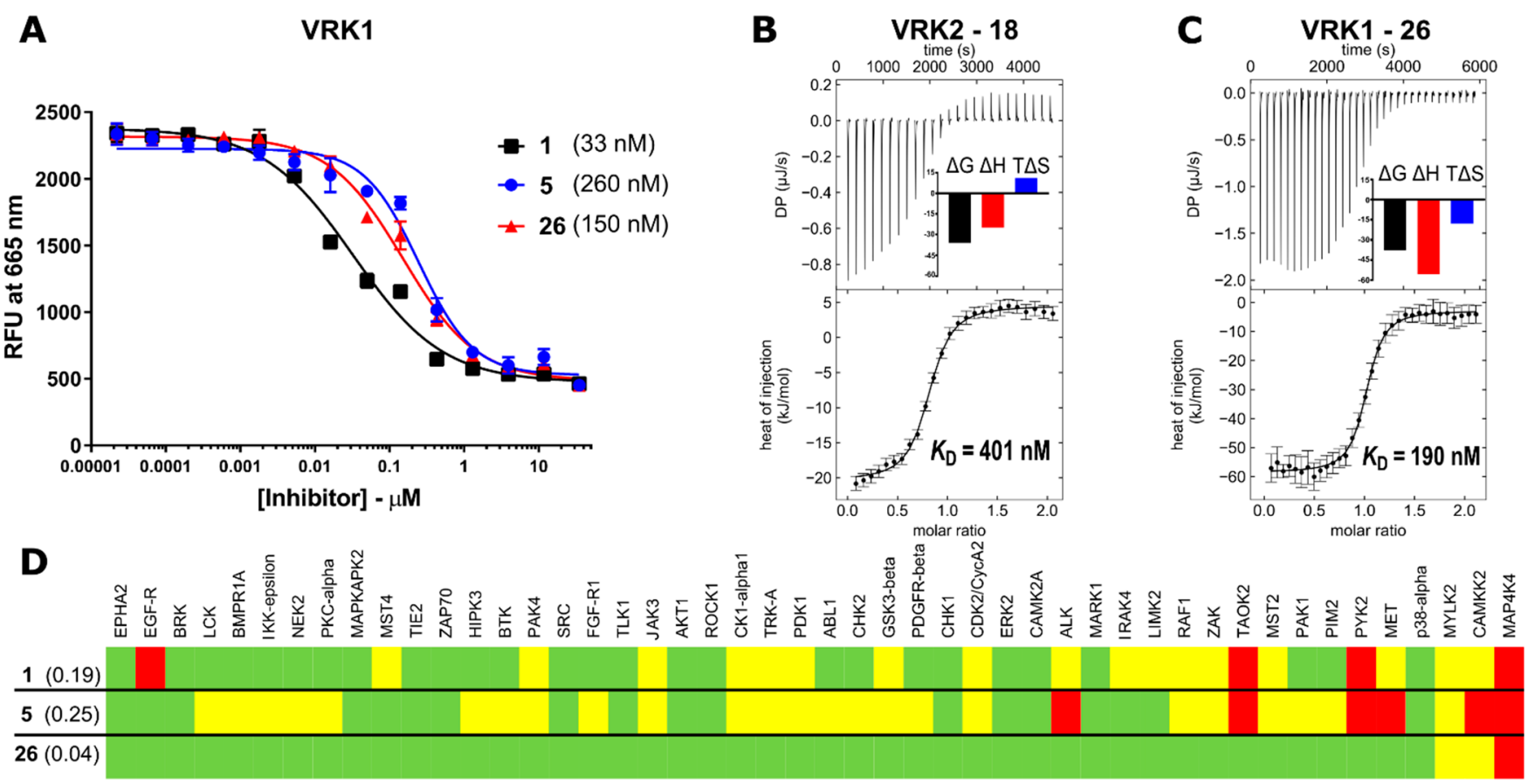

Figure 2. Biochemical, biophysical, and selectivity profile characterization of key compounds. (A) $\mathrm{IC}_{50}$ determination curves for $\mathbf{1}, \mathbf{5}$, and $\mathbf{2 6}$ against VRK1-FL. Individual data points are the mean \pm SD of three technical replicates. Estimated $\mathrm{IC}_{50}$ values are shown in parentheses. (B,C) ITC measurements for top compounds in the DSF assay for VRK2-KD (18) and VRK1-FL (26). Values for binding energies are shown in $\mathrm{kJ} / \mathrm{mol}$. (D) Selectivity profile of 1 (BI-D1870), 5, and 26 against a representative panel of 48 human kinases at $1 \mu \mathrm{M}$ concentration. Colors denote \% residual kinase activity: green, residual activity $>80 \%$; yellow, residual activity $\geq 40 \%$ and $\leq 80 \%$; red, residual activity $<40 \%$. Residual activity values are shown in Supplementary Table S5. Numbers in parentheses report the selectivity score S(50\%) for each compound. S(50\%) was obtained by dividing the number of protein kinases having $\%$ residual activity $\leq 50 \%$, by the total number of tested protein kinases. Distribution of selected kinases across the human kinome is shown in Supplementary Figure S7. The VRKs were not part of the panel.
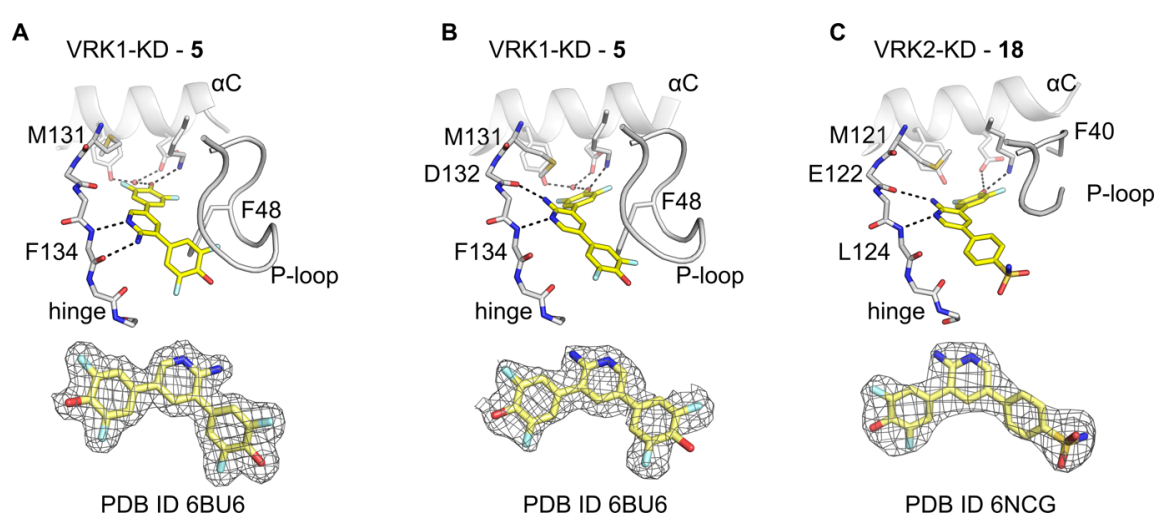

Figure 3. Cocrystal structures revealed distinct compound binding modes. (A,B) VRK1-KD bound to 5. The compound adopts two different binding modes with different VRK1 molecules in the crystal. (C) VRK2-KD bound to 18. Black dashed lines represent potential hydrogen bonds. Bottom panels show electron density maps $\left(2 F_{o}-F_{c}\right)$ contoured at $1.0 \sigma$.

Although robust, DSF provides only limited information on compound binding to a target protein. We then developed an enzyme inhibition assay (Supplementary Figure S2) and determined the potency $\left(\mathrm{IC}_{50}\right)$ of key compounds against VRK1-FL (Table 1, Figure 2, Supplementary Table S2). In these assays, our prototype compound 5 had an $\mathrm{IC}_{50}$ value of $260 \mathrm{nM}, \sim 8$-fold higher than the one observed for 1 (BID1870, $33 \mathrm{nM}$ ). Estimated $\mathrm{IC}_{50}$ values were in good agreement with our DSF results. For example, 19, with a $\Delta T_{\mathrm{m}}$ of $2.2{ }^{\circ} \mathrm{C}$, had an $\mathrm{IC}_{50}$ of $674 \mathrm{nM}$ against VRK1-FL (Supplementary Figure S3). More importantly, our enzymatic assays confirmed these compounds as inhibitors of VRK1.
Unfortunately, we did not succeed in developing a similar enzymatic assay for VRK2. Thus, we employed isothermal titration calorimetry (ITC) to estimate binding potency $\left(K_{\mathrm{D}}\right)$ of selected compounds to VRK2-KD, including 18, our top compound from the DSF assay (Figure 2, Supplementary Figure S4). Our ITC data confirmed binding of these compounds to VRK2-KD and revealed these interactions to be mostly enthalpy-driven $(|\Delta H|>|T \Delta S|$ ) (Supplementary Table S3). As expected, 18 had the strongest binding to VRK2 $\left(K_{\mathrm{D}} \approx 400 \mathrm{nM}\right)$. At the moment, we cannot offer an explanation for the discrepancy between ITC and DSF results for 13 and 19. 
To better understand the molecular basis for ligand binding, we obtained cocrystals of VRK1-KD (residues Arg3-Glu364) bound to 5 and VRK2-KD bound to 18 (Figure 3). Both structures were solved by molecular replacement (Supplementary Table S4). Ligand-bound VRK1-KD and VRK2-KD adopted an active conformation, in which conserved residues from structurally conserved elements of the kinase domain (Glu83 and Lys71 within $\alpha \mathrm{C}$-helix and the ATP-binding site, respectively, VRK1 numbering) are brought into close proximity, and the side chain of Asp197 (VRK1 numbering) from the conserved DFG motif (DYG in the VRKs) points toward the ATP-binding site (Figure 3 and Supplementary Figure S5A,B).

As expected, $\mathbf{5}$ and $\mathbf{1 8}$ were found in the ATP-binding sites of VRK1 and VRK2, respectively (Figure 3A,B). The binding pose for 18 showed the 2 -amino moiety pointed toward the back of VRK2 ATP-binding site. The 2-amino group and the pyridine $\mathrm{N}$ atom of $\mathbf{1 8}$ established one hydrogen bond each to the carbonyl and amide groups of VRK2 hinge residues Glu122 and Leu124, respectively. In VRK1-KD crystals, the ligand could be observed in three out of the four protein molecules in the asymmetric unit and, surprisingly, was found in two different poses. The first of these was equivalent to the one observed for 18 bound to VRK2-KD. In the second binding mode, the 2 -amino group of 5 pointed toward the solvent and, together with the pyridine nitrogen atom, facilitated HBs with main chain atoms from VRK1-KD hinge residue Phe134.

The cocrystal structures helped us to rationalize the relevance of the difluorophenol moiety for binding. Regardless of compound binding pose, this group facilitated a HB network with polar side chains from structurally conserved residues within the kinase domain of VRK1 (Lys71 and Glu83) and VRK2 (Lys61 and Glu73). The difluorophenol group participating in these contacts displayed distinct dihedral angles to the 2 -amino core depending on its attachment position: $\sim 45^{\circ}$ in $\mathrm{R}^{1}$ and $\sim 9^{\circ}$ in $\mathrm{R}^{2}$. In VRK1, these different orientations of the difluorophenol group were accommodated by a corresponding movement of the side chain from residue Met131, which occupies the gatekeeper position in this protein. Consequently, the difluorophenol group fitted tightly between the $\alpha \mathrm{C}$-helix and the gatekeeper residue in both poses. These observations might explain why we could not find substituents that improved binding over the difluorophenol group.

The VRK2-KD cocrystal structure also revealed that the $\mathbf{1 8}$ sulfonamide group pointed away from the protein ATPbinding site and was mostly solvent-exposed. A similar observation was made for the difluorophenol group in $\mathbf{5}$ that did not interact with VRK1-KD $\alpha$ C-helix (Supplementary Figure S5D-F). Our DSF results also indicated that placement of polar groups in the meta-position resulted in slight increases of $\Delta T_{\mathrm{m}}$, especially for VRK2-KD (10 vs 11, for example). At this position, polar groups from the ligand might be able to engage polar groups from VRK2-KD P-loop.

Regardless of the ligand binding pose, the P-loop of VRK1 was found to be folded over 5. This conformation was likely stabilized by hydrophobic interactions observed between Ploop residue Phe48 and 5's three-ring system. By contrast, VRK2 P-loop did not fold over 18. In our VRK2 cocrystal, the P-loop was found rotated toward the protein $\alpha \mathrm{C}$-helix by $\sim 6 \AA$ (Supplementary Figure S5C). Consequently, equivalent aromatic residues within the P-loop of VRK1 (Phe48) and
VRK2 (Phe40) occupied different positions in each of the proteins' ATP-binding site.

The two binding modes observed for $\mathbf{5}$ in VRK1 suggested that the 2-amino moiety had no binding preference for either of the hinge carbonyl groups it can interact with (Figure 3A,B). This led us to hypothesize that these two interactions were either equally productive or equally weak in the binding process. To address these hypotheses, we synthesized the following analogues: (i) 23, with two amino groups that could interact with both hinge carbonyl groups simultaneously; (ii) 24, with a 2 -amino and a space-filling 6-methyl group; (iii) 25, with the 2-amino group removed; and (iv) 26, with the 2 amino group substituted by a 2-methyl group (Table 1, Supplementary Table S1).

DSF assays revealed that none of these new analogs had improved $\Delta T_{\mathrm{m}}$ values for VRK2-KD (Table 1, Supplementary Table S1). These results suggested that the HB between the hinge carbonyl group and the 2-aminopyridine core is a productive interaction for VRK2. Likewise, for VRK1-FL, compounds 23, 24, and 25 did not improve $\Delta T_{\mathrm{m}}$ values over those observed for 5. Poor results observed for 23 and 24 might be explained by clashes between one of the two substituents in these compounds (at the 2- or 6-position in the pyridine core) and main chain atoms from residues within the kinase hinge region.

By contrast, 26 and 5 were equipotent in the DSF assay, supporting the hypothesis that the 2-amino moiety contributed little to the binding of 5 to VRK1. Thus, both our DSF results and crystal structures suggested that VRK1, but not VRK2, was capable of binding equally well to the 2 -aminopyridine core in two different conformations.

Further, the increase in $\Delta T_{\mathrm{m}}$ observed for 26 (2methylpyridine core) over 25 (pyridine core) for VRK1 might arise from favorable electron-donating properties or conformational effects conferred to the pyridine ring system by the presence of an adjacent methyl group. ${ }^{23,24}$ Alternatively, the lack of a substituent group in the 2-position may incur a free energy penalty associated with 25 's inability to fill in a cavity in the protein binding pocket.

Enzyme inhibition assays further confirmed 26 as a VRK1 inhibitor with an $\mathrm{IC}_{50}$ value of $\sim 150 \mathrm{nM}$, a $\sim 2$-fold increase in potency over prototype compound $\mathbf{5}$ (Figure 1A, Table1). ITC experiments determined that binding of $\mathbf{2 6}$ to VRK1-FL was enthalpy-driven and had a $K_{\mathrm{D}}$ of $190 \mathrm{nM}$ (Figure 1C, Supplementary Table S3).

The 2-amino-pyridine moiety is a common feature of many kinase inhibitors and often plays a role in binding to the kinase hinge region (Supplementary Figure S6). ${ }^{25}$ We were surprised that changes in hinge-binding groups within the pyridine core had such marked impact on compound binding to each of the VRKs, as these proteins have highly similar ATP-binding sites. Motivated by these findings, we assessed the activity of 1, 5, and 26 against a representative panel of 48 human kinases at 1 $\mu \mathrm{M}$ concentration (Figure 2D, Supplementary Figure S7). Our results showed that introduction of the methyl group in the pyridine scaffold markedly improved selectivity of 26 over prototype 5; S(50\%) scores of 0.04 and 0.25 , respectively. Our selectivity data also confirmed previous reports that 1 (BID1870) is fairly promiscuous; ${ }^{17} \mathrm{~S}(50 \%)$ score of 0.19 .

To ascertain that $\mathbf{1 8}$ and $\mathbf{2 6}$ represented tractable molecules for development into chemical probes suitable for biological studies, we performed cell viability assays based on the metabolic conversion of a tetrazolium dye (MTT, see 
Supporting Information) to its insoluble formazan by human (HeLa) cells. Our results indicated that there was no noticeable effect on cell viability after a 24 -h treatment with $3.2 \mu \mathrm{M}$ of 18 and only a slight decrease with $3.2 \mu \mathrm{M}$ of 26 ; and $\geq 60 \%$ of cells remained viable after the compound concentration was raised to $12.5 \mu \mathrm{M}$ of 18 and $25 \mu \mathrm{M}$ of 26 (Supplementary Figure S8).

In conclusion, we report here the use of the pyridine scaffold for the development of inhibitors against VRK1 and VRK2. Our data revealed 26 to be a selective $(\mathrm{S}(50 \%) 0.04)$ and potent $\left(\mathrm{IC}_{50} \approx 150 \mathrm{nM}\right)$ inhibitor of VRK1. Compound 18 binds to VRK2 in the mid-nM range (ITC $K_{\mathrm{D}} 400 \mathrm{nM}$ ). Further characterization of this compound is likely to confirm 18 as an inhibitor of the VRK2 protein. Both compounds are not overtly toxic to human cells and represent promising starting points for developing more potent and selective inhibitors for VRK1 and VRK2. Future optimization efforts will take advantage of the scaffold's good synthetic accessibility.

\section{ASSOCIATED CONTENT}

\section{S Supporting Information}

The Supporting Information is available free of charge on the ACS Publications website at DOI: 10.1021/acsmedchemlett.9b00082.

Supplementary Tables S1-S5, Supplementary Figures S1-S8, Supplementary Methods, and Supplementary References (PDF)

\section{Accession Codes}

The atomic coordinates and crystallographic structures factors for VRK1-KD bound to 5 and for VRK2-KD bound to 18 have been deposited in the Protein Data Bank (www.rcsb.org) with accession codes 6BU6 and 6NCG, respectively.

\section{AUTHOR INFORMATION}

\section{Corresponding Authors}

*E-mail: rafael.counago@unicamp.br.

*E-mail: cristiano.guimaraes@ache.com.br.

\section{ORCID $\odot$}

Rafael M. Couñago: 0000-0003-1847-5090

\section{Author Contributions}

\# These authors contributed equally. R.A.M.S., F.H.S.G., S.N.S.V., H.A., A.M., and C.R.W.G. designed all molecules. R.A.M.S., F.H.S.G., and S.N.S.V. synthesized and characterized all molecules. C.V.R. and R.M.C. designed and performed crystallization experiments, X-ray diffraction data collection, and protein structure determination and analysis. A.S.S. designed, performed, and analyzed ITC experiments for VRK2-KD. J.E.T. designed, performed, and analyzed ITC experiments for VRK1-FL. C.V.R., A.S.S., and L.A.D. designed, performed, and analyzed DSF experiments. L.A.D. designed, performed, and analyzed enzymatic assays. F.D.P. designed, performed, and analyzed cell assays. H.A., A.M., J.M.E., K.B.M., O.G., C.R.W.G., and R.M.C. coordinated the project. R.A.M.S. and R.M.C. wrote the manuscript. All authors revised the manuscript. All authors have given approval to the final version of the manuscript.

\section{Funding}

This work was supported by the Brazilian agencies FAPESP (Fundação de Amparo à Pesquisa do Estado de São Paulo) (2013/50724-5 and 2014/5087-0), Embrapii (Empresa Brasileira de Pesquisa e Inovação Industrial), and $\mathrm{CNPq}$
(Conselho Nacional de Desenvolvimento Científico e Tecnológico) (465651/2014-3 and 400906/2014-7). The SGC is a registered charity (number 1097737) that receives funds from AbbVie, Bayer Pharma AG, Boehringer Ingelheim, Canada Foundation for Innovation, Eshelman Institute for Innovation, Genome Canada, Innovative Medicines Initiative (EU/EFPIA) [ULTRA-DD grant no. 115766], Janssen, Merck KGaA Darmstadt Germany, MSD, Novartis Pharma AG, Ontario Ministry of Economic Development and Innovation, Pfizer, Takeda, and Wellcome [106169/ZZ14/Z]. R.A.M.S., S.N.S.V. and F.D.P. received FAPESP fellowships (2016/ 25320-6, 2018/09475-5 and 2018/03359-3 respectively). C.V.R. and A.S.S. received CAPES (Coordenação de Aperfeiçoamento de Pessoal de Nível Superior) postdoctoral fellowships (88887.146077/2017-00 and 88887.136342/201700 , respectively).

\section{Notes}

The authors declare the following competing financial interest(s): The authors F.H.S.G., H.A., A.M., C.R.W.G. are employed by Aché Laboratórios Farmacêuticos. All other authors declare no competing financial interest.

\section{ACKNOWLEDGMENTS}

We thank the staff of the Proteomics section of the Life Sciences Core Facility (LaCTAD), part of the University of Campinas (UNICAMP), for their contributions to ITC analysis. We thank the staff at the Northeastern Collaborative Access Team beamlines (GU56413 and GU54127), which are funded by the National Institute of General Medical Sciences from the National Institutes of Health (P41 GM103403). The Pilatus $6 \mathrm{M}$ detector on the 24-ID-C beamline is funded by a NIH-ORIP HEI grant (S10 RR029205). This research used resources of the Advanced Photon Source, a U.S. Department of Energy (DOE) Office of Science User Facility operated for the DOE Office of Science by Argonne National Laboratory under Contract No. DE-AC02-06CH11357.

\section{ABBREVIATIONS}

VRK, Vaccinia-related kinase; KD, kinase domain; FL, fulllength; HB, hydrogen bond; MTT, 3-(4,5-dimetrylthiazol-2yl)-2,5-diphenyltetrazolium bromide

\section{REFERENCES}

(1) Nichols, R. J.; Traktman, P. Characterization of Three Paralogous Members of the Mammalian Vaccinia Related Kinase Family. J. Biol. Chem. 2004, 279 (9), 7934.

(2) Scheeff, E. D.; Eswaran, J.; Bunkoczi, G.; Knapp, S.; Manning, G. Structure of the Pseudokinase VRK3 Reveals a Degraded Catalytic Site, a Highly Conserved Kinase Fold, and a Putative Regulatory Binding Site. Structure 2009, 17 (1), 128.

(3) Kang, T.-H.; Park, D.-Y.; Choi, Y. H.; Kim, K.-J.; Yoon, H. S.; Kim, K.-T. Mitotic Histone H3 Phosphorylation by Vaccinia-Related Kinase 1 in Mammalian Cells. Mol. Cell. Biol. 2007, 27 (24), 8533.

(4) Molitor, T. P.; Traktman, P. Depletion of the Protein Kinase VRK1 Disrupts Nuclear Envelope Morphology and Leads to BAF Retention on Mitotic Chromosomes. Mol. Biol. Cell 2014, 25 (6), 891.

(5) Monsalve, D. M.; Merced, T.; Fernández, I. F.; Blanco, S.; Vázquez-Cedeira, M.; Lazo, P. A. Human VRK2Modulates Apoptosis by Interaction with Bcl-XL and Regulation of BAX Gene Expression. Cell Death Dis. 2013, 4 (2), No. e513.

(6) Blanco, S.; Klimcakova, L.; Vega, F. M.; Lazo, P. A. The Subcellular Localization of Vaccinia-Related Kinase-2 (VRK2) 
Isoforms Determines Their Different Effect on P53 Stability in Tumour Cell Lines. FEBS J. 2006, 273 (11), 2487.

(7) Li, M.; Wang, Y.; Zheng, X. B.; Ikeda, M.; Iwata, N.; Luo, X. J.; Chong, S. A.; Lee, J.; Rietschel, M.; Zhang, F.; et al. Meta-Analysis and Brain Imaging Data Support the Involvement of VRK2 (Rs2312147) in Schizophrenia Susceptibility. Schizophr. Res. 2012, 142 (1-3), 200.

(8) Chang, H.; Zhang, C.; Xiao, X.; Pu, X.; Liu, Z.; Wu, L.; Li, M. Further Evidence of VRK2 Rs2312147 Associated with Schizophrenia. World J. Biol. Psychiatry 2016, 17 (6), 457.

(9) Azimi, T.; Ghafouri-Fard, S.; Davood Omrani, M.; Mazdeh, M.; Arsang-Jang, S.; Sayad, A.; Taheri, M. Vaccinia Related Kinase 2 (VRK2) Expression in Neurological Disorders: Schizophrenia, Epilepsy and Multiple Sclerosis. Mult. Scler. Relat. Disord. 2018, 19, 15.

(10) Li, M.; Yue, W. VRK2, a Candidate Gene for Psychiatric and Neurological Disorders. Mol. Neuropsychiatry 2018, 4 (3), 119.

(11) Jeong, Y.-H.; Choi, J.-H.; Lee, D.; Kim, S.; Kim, K.-T. VacciniaRelated Kinase 2 Modulates Role of Dysbindin by Regulating Protein Stability. J. Neurochem. 2018, 147 (5), 609.

(12) Huang, W.; Cui, X.; Chen, Y.; Shao, M.; Shao, X.; Shen, Y.; Liu, Q.; Wu, M.; Liu, J.; Ni, W.; et al. High VRK1 Expression Contributes to Cell Proliferation and Survival in Hepatocellular Carcinoma. Pathol., Res. Pract. 2016, 212 (3), 171.

(13) Ben, Z.; Gong, L.; Qiu, Y. High Expression of VRK1 Is Related to Poor Prognosis in Glioma. Pathol., Res. Pract. 2018, 214 (1), 112.

(14) Elkins, J. M.; Fedele, V.; Szklarz, M.; Abdul Azeez, K. R.; Salah, E.; Mikolajczyk, J.; Romanov, S.; Sepetov, N.; Huang, X. P.; Roth, B. L.; et al. Comprehensive Characterization of the Published Kinase Inhibitor Set. Nat. Biotechnol. 2016, 34 (1), 95.

(15) Couñago, R. M.; Allerston, C. K.; Savitsky, P.; Azevedo, H.; Godoi, P. H.; Wells, C. I.; Mascarello, A.; de Souza Gama, F. H.; Massirer, K. B.; Zuercher, W. J.; et al. Structural Characterization of Human Vaccinia-Related Kinases (VRK) Bound to Small-Molecule Inhibitors Identifies Different P-Loop Conformations. Sci. Rep. 2017, 7 (1), 7501.

(16) Guimarães, C. R. W.; Rai, B. K.; Munchhof, M. J.; Liu, S.; Wang, J.; Bhattacharya, S. K.; Buckbinder, L. Understanding the Impact of the P-Loop Conformation on Kinase Selectivity. J. Chem. Inf. Model. 2011, 51 (6), 1199.

(17) Aronchik, I.; Appleton, B. A.; Basham, S. E.; Crawford, K.; Del Rosario, M.; Doyle, L. V.; Estacio, W. F.; Lan, J.; Lindvall, M. K.; Luu, C. A.; et al. Novel Potent and Selective Inhibitors of P90 Ribosomal S6 Kinase Reveal the Heterogeneity of RSK Function in MAPKDriven Cancers. Mol. Cancer Res. 2014, 12 (5), 803.

(18) Ammirati, M.; Bagley, S. W.; Bhattacharya, S. K.; Buckbinder, L.; Carlo, A. A.; Conrad, R.; Cortes, C.; Dow, R. L.; Dowling, M. S.; El-Kattan, A.; et al. Discovery of an in Vivo Tool to Establish Proofof-Concept for MAP4K4-Based Antidiabetic Treatment. ACS Med. Chem. Lett. 2015, 6 (11), 1128.

(19) Fedorov, O.; Niesen, F. H.; Knapp, S. Kinase Inhibitor Selectivity Profiling Using Differential Scanning Fluorimetry. Methods Mol. Biol. 2012, 795, 109.

(20) Fedorov, O.; Marsden, B.; Pogacic, V.; Rellos, P.; Muller, S.; Bullock, A. N.; Schwaller, J.; Sundstrom, M.; Knapp, S. A Systematic Interaction Map of Validated Kinase Inhibitors with Ser/Thr Kinases. Proc. Natl. Acad. Sci. U. S. A. 2007, 104 (51), 20523.

(21) Qiu, J.; Stevenson, S. H.; O’Beirn, M. J.; Silverman, R. B. 2,6Difluorophenol as a Bioisostere of a Carboxylic Acid: Bioisosteric Analogues of Gamma-Aminobutyric Acid. J. Med. Chem. 1999, 42 (2), 329.

(22) Jain, R.; Mathur, M.; Lan, J.; Costales, A.; Atallah, G.; Ramurthy, S.; Subramanian, S.; Setti, L.; Feucht, P.; Warne, B.; et al. Discovery of Potent and Selective RSK Inhibitors as Biological Probes. J. Med. Chem. 2015, 58 (17), 6766.

(23) Barreiro, E. J.; Kummerle, A. E.; Fraga, C. A. M. The Methylation Effect in Medicinal Chemistry. Chem. Rev. 2011, 111 (9), 5215.
(24) Leung, C. S.; Leung, S. S. F.; Tirado-Rives, J.; Jorgensen, W. L. Methyl Effects on Protein-Ligand Binding. J. Med. Chem. 2012, 55 (9), 4489.

(25) Xing, L.; Klug-Mcleod, J.; Rai, B.; Lunney, E. A. Kinase Hinge Binding Scaffolds and Their Hydrogen Bond Patterns. Bioorg. Med. Chem. 2015, 23 (19), 6520. 